\title{
TENDÊNCIAS METEOROLÓGICAS E ANOMALIAS DE EVAPOTRANSPIRAÇÃO DE REFERÊNCIA NO PERÍODO DE 2011-2017 PARA O ESTADO DE MINAS GERAIS
}

\author{
ALMEIDA, Lucas Rosa de - lucasalmeidacristais@hotmail.com \\ Universidade Federal de Lavras / UFLA
}

\author{
ROSSE, Vinicius Possato - viniciuspossato12@gmail.com \\ Universidade Federal de Lavras / UFLA
}

VIOLA, Marcelo Ribeiro - marcelo.viola@ufla.br

Universidade Federal de Lavras / UFLA

VIEIRA-FILHO, Marcelo - marcelo.filho@ufla.br

Universidade Federal de Lavras / UFLA

\begin{abstract}
RESUMO: Dentre as componentes necessárias à realização do balanço hídrico climatológico, encontra-se a evapotranspiração de referência. Esta, refere-se às saídas de água do solo e tradicionalmente é calculada por diferentes métodos indiretos, a partir de observações realizadas nas estações meteorológicas. Neste trabalho, informações de 27 estações meteorológicas convencionais localizadas no estado de Minas Gerais foram avaliadas, objetivando-se à identificação de tendências climatológicas e ao estudo das anomalias de evapotranspiração no período de 2011-2017, levando-se em consideração o período de 1981-2010 (Normal climatológica). Foi utilizado o método de PenmanMonteith para o cálculo da evapotranspiração de referência pelo software REF-ET. Além disso, também foi utilizada a programação em ambiente $R$, por meio da qual os dados meteorológicos foram manipulados e os testes estatísticos foram realizados. Para a verificação das tendências não sazonais, o método de Mann-Kendall foi o utilizado. A análise da tendência para temperatura (máxima, mínima e média compensada), insolação e velocidade do vento mostrou tendências de aumento significativas (valor de $p$ $<0,05)$, enquanto que, para a umidade relativa foi obtida tendência de redução. Para as estações localizadas na região Norte do Estado de Minas Gerais foram observadas anomalias positivas de evapotranspiração de maneira consistente ao longo de todo o ano. A estação de Pedra Azul, localizado na região do Jequitinhonha, apresentou anomalia positiva de evapotranspiração em todos os meses com valores acima de $20 \mathrm{~mm}$. mês ${ }^{-1}$. Além disso, anomalias positivas persistentes de evapotranspiração foram observadas no trimestre DJF (dezembro, janeiro e fevereiro) acumulando valores acima de $40 \mathrm{~mm}$. mês ${ }^{-1}$ para as estações de Juiz de Fora, Janaúba, Lavras, Araxá e Montes Claros. Tais anomalias mostram que o período de 2011 a 2017 apresentou clima mais quente e seco que a normal climatológica (1981-2010).
\end{abstract}

PALAVRAS-CHAVE: evapotranspiração de referência, tendências climatológicas, Penman-Monteith, Mann-Kendall, mudanças climáticas, anomalias.

\section{METEOROLOGICAL TRENDS AND REFERENCE EVAPOTRANSPIRATION ANOMALIES IN THE} PERIOD 2011-2017 FOR THE STATE OF MINAS GERAIS

ABSTRACT: Among the components necessary to perform the climatological water balance, is the reference evapotranspiration. This, refers to the outflow of water in the soil and is traditionally calculated by different indirect methods, based on observations made in the meteorological stations. In this work, information from 27 conventional weather stations located in the state of Minas Gerais was evaluated, aiming at the identification of climatological trends and the study of evapotranspiration anomalies in the period 2011-2017, taking into account the period of 1981- 2010 (Normal Climatological). The Penman-Monteith method was used to calculate the reference evapotranspiration by REF-ET software. In addition, it was also used the programming in 
$\mathrm{R}$ environment, by means of which the meteorological data were manipulated and the statistical tests were carried out. For the verification of non-seasonal trends, Kendall's method was used. The trend of temperature (maximum, minimum and average compensated), insolation and wind speed showed significant increase tendencies ( $p$ value $<0.05$ ), while for the relative humidity a tendency of reduction was obtained. For the stations inserted in the Northern region of the State of Minas Gerais, positive evapotranspiration anomalies were observed consistently throughout the year. The weather stations of Pedra Azul, located in the Jequitinhonha region, presented a positive evapotranspiration anomaly in all months with values above $20 \mathrm{~mm} \cdot \mathrm{m}^{-1}$. In addition, persistent positive anomalies of evapotranspiration were observed in the DJF quarter (December, January and February) accumulating values above $40 \mathrm{~mm}$. month ${ }^{-1}$ for the stations of Juiz de Fora, Janaúba, Lavras, Araxá and Montes Claros. Such anomalies show that the period from 2011 to 2017 presented a warmer and drier climate than the climatological normal (1981-2010).

KEYWORDS: Reference evapotranspiration, climatic trends, Penman-Monteith, Mann-Kendall, climate change, anomalies.

\section{TENDENCIAS METEOROLÓGICAS Y ANOMALÍAS DE EVAPOTRANSPIRACIÓN DE REFERENCIA EN EL PERÍODO 2011-2017 PARA EL ESTADO DE MINAS GERAIS}

RESUMEN: Entre los componentes necesarios para la realización del balance hídrico climatológico, se encuentra la evapotranspiración de referencia. Esta se refiere a las salidas de agua en el suelo y tradicionalmente se calcula por diferentes métodos indirectos, a partir de observaciones realizadas en las estaciones meteorológicas. En este trabajo, informaciones de 27 estaciones meteorológicas convencionales localizadas en el estado de Minas Gerais fueron evaluadas, objetivándose a la identificación de tendencias climatológicas y al estudio de las anomalías de evapotranspiración en el período de 20112017, teniendo en cuenta el período de 1981- 2010 (Normal Climatológico). Se utilizó el método de Penman-Monteith para el cálculo de la evapotranspiración de referencia por el software REF-ET. Además, también se utilizó la programación en ambiente $R$, por medio de la cual los datos meteorológicos fueron manipulados y las pruebas estadísticas se realizaron. Para la verificación de las tendencias no estacionales, el método de Kendall fue el utilizado. El análisis de la tendencia a la temperatura (máxima, mínima y media compensada), insolación y velocidad del viento mostró tendencias de aumento significativas (valor de $\mathrm{p}<0,05$ ), mientras que para la humedad relativa se obtuvo una tendencia de reducción. Para las estaciones insertadas en la región Norte del Estado de Minas Gerais se observaron anomalías positivas de evapotranspiración de manera consistente a lo largo de todo el año. La estación meteorológica de Pedra Azul, localizado en la región del Jequitinhonha, presentó una anomalía positiva de evapotranspiración en todos los meses con valores superiores a $20 \mathrm{~mm}$.més ${ }^{-1}$. Además, las anomalías positivas persistentes de evapotranspiración se observaron en el trimestre del DJF (diciembre, enero y febrero) acumulando valores por encima de $40 \mathrm{~mm}$. més ${ }^{-1}$ para las estaciones de Juiz de Fora, Janaúba, Lavras, Araxá y Montes Claros. Tales anomalías muestran que el período de 2011 a 2017 presentó un clima más cálido y seco que la normal climatológica (1981-2010).

PALABRAS CLAVE: Evapotranspiración de referencia, tendencias climáticas, Penman-Monteith, Mann-Kendall, cambio climático, anomalías.

\section{INTRODUÇÃO}

Dentre as componentes utilizadas para a determinação da presença de água no solo, encontra-se a evapotranspiração, termo que descreve a saída da água à atmosfera em forma de vapor d'água como resultado dos processos simultâneos de evaporação da superfície e de transpiração das plantas (CAMARGO E CAMARGO, 2000; DENSKI E BACK, 2015; SENTELHAS, GILLESPIE 
E SANTOS, 2010). Tal mecanismo é classificado na literatura em evapotranspiração de referência e em evapotranspiração real (BEZERRA, SILVA E FERREIRA, 2008; CARVALHO et al., 2011). O primeiro descreve a perda de água de uma área cultivada com grama, com altura de 0,08 a $0.15 \mathrm{~m}$ e ausência de estresse hídrico para atender às necessidades da evaporação do solo e da transpiração; o segundo descreve a perda de água à atmosfera em qualquer ambiente natural e para quaisquer condições (CARVALHO et al., 2011; THORNTHWAITE, 1948).

A importância de avaliar a disponibilidade de água no solo ocorre em virtude de sua complexidade e interdisciplinaridade. Dentre esses desafios, cabe citar a perda hídrica pela evapotranspiração (ALENCAR et al., 2011). Dentre as áreas de conhecimento que buscam a melhoria contínua na representação dos processos de evapotranspiração, citam-se: irrigação (FRANCISCO E PINTO, 2012; FREITAS, BEZERRA E FONTENELE, 1999), hidrologia (GOMES, 2005) e ciências florestais (MOSTER et al., 2003). No entanto, para que tais aplicações logrem êxito, o conhecimento dos fatores intervenientes ao processo é condição imprescindível. De forma geral, a componente da evapotranspiração é afetuada diretamente pela radiação solar, temperatura do ar, intensidade do vento, tipo de cultura e pressão de vapor (ALENCAR, SEDIYAMA E MANTOVANI, 2015; ALLEN et al., 1998).

Para a estimativa da evapotranspiração de referência, existem diversos métodos indiretos que podem ser utilizados. De forma geral, esses métodos se dão por meio da junção de dados meteorológicos e aplicação em equações (MENDES, 2006). Dentre os mais citados na literatura específica, encontram-se: Thornthwaite (THORNTHWAITE, 1948), Hargreaves (LIMA, et al., 2016), Penmann-Monteith (ALLEN et al., 1998), por exemplo. Dentre os métodos citados, o método de Penmann-Monteith é considerado como referência, por abranger o maior número de variáveis; além disso, é adotado como padrão pela FAO (Organização das Nações Unidas para Alimentação e Agricultura). Atualmente, por meio do avanço computacional, há possibilidade de se estimar a evapotranspiração por meio do software REF-ET (ALLEN, 2013).

Uma grande preocupação atual refere-se às mudanças climáticas. Resultados de pesquisas recentes demonstram que mudanças climáticas podem acarretar consequências negativas, sobretudo a intensificação de eventos extremos, como desertificação (ALMEIDA et al., 2014), e aumento de temperatura (NATIVIDADE, GARCIA E TORRES, 2017), entre outros. Segundo projeções, é previsto um aumento da temperatura média global entre $1,8^{\circ} \mathrm{C}$ e $4,0^{\circ} \mathrm{C}$ até $\mathrm{o}$ ano de 2100 ; tratando-se do Brasil, espera-se que esse aumento varie de $3,0^{\circ} \mathrm{C}$ a $4,0^{\circ} \mathrm{C}$ (ÁVILA, 2007; BLANK, 2015; IPCC, 2014). Dessa forma, as condições de cobertura vegetal, umidade e temperatura poderão se alterar, com impactos sobre a evapotranspiração. Alguns estudos relacionados discutem tais implicações (KINGSTON et al., 2009; REMROVA E CÍSLEROVÁ, 2010; WANG, XU e SUN, 2006).

O presente estudo concentra-se na área do estado de Minas Gerais, cuja empregabilidade do setor de agricultura é maior que o setor secundário (BASTOS E GOMES, 2011) e se encontra em constante modernização. Entretanto, no que se refere à disponibilidade hídrica, esta vem sendo objeto de estudos devido aos recentes períodos de escassez hídrica (FAYER et al., 2017), o que tem motivado o desenvolvimento de pesquisas acerca das variáveis 
temperatura, precipitação e evapotranspiração (LEMOS FILHO et al., 2010). Diante disso, objetivou-se analisar as anomalias de evapotranspiração do período de 2011 a 2017 em comparação com o período da série histórica de 1981 a 2010, e a presença de tendência para a insolação, velocidade do vento, umidade relativa e temperaturas (máxima, média compensada e mínima) para o Estado de Minas Gerais.

\section{MATERIAIS E MÉTODOS}

O presente trabalho concentrou-se no estado de Minas Gerais, localizado na região sudeste do Brasil e que apresenta uma extensão territorial de 586.528 $\mathrm{km}^{2}$. Segundo estimativa do IBGE (2018), a população de Minas Gerais é de, aproximadamente, 21 milhões de habitantes. Tratando-se da vegetação local, o estado é coberto por, praticamente, Cerrado (oeste do estado) - composto por arbustos e gramíneas - e Mata Atlântica (leste do estado) - sendo caracterizada por uma densa vegetação, elevado índice de precipitação e grande biodiversidade (COURA, 2007; PENEREIRO et al., 2018). Em relação às características climáticas, segundo a classificação de Köopen, utilizada na classificação de zonas homogêneas através das variáveis de precipitação e temperatura (JÚNIOR, 2009; KÖPPEN, 2011; KOTTEK et al., 2006), o estado de Minas, para o clima presente (1981-2010), apresenta as seguintes classificações: Cwa e Cwb (temperados), Bsh e BWh (áridos), Aw (tropical) (REBOITA et al., 2017), e ainda, Cfa e Cfb (ALVARES et al., 2014)

\subsection{DADOS METEOROLÓGICOS}

Nesse estudo utilizaram-se os dados meteorológicos de 27 estações de superfície oriundos do Banco de Dados Meteorológicos para Ensino e Pesquisa (BDMEP) fornecidos pelo Instituto Nacional de Meteorologia (INMET) e localizados no endereço eletrônico: http://www.inmet.gov.br/projetos/rede/pesquisa/.

A figura 1 apresenta a distribuição espacial das estações meteorológicas. Os dados meteorológicos referentes a temperatura máxima, mínima e compensada, além da intensidade do vento, insolação e umidade relativa de 1981 a 2017 foram utilizados para as estimativas da evapotranspiração de referência e cálculos de tendência. As análises foram realizadas para dois períodos: (i) normal climatológica do período de 1981 a 2010, considerado referência; e (ii) o período recente de 2011 a 2017.

Uma vez que os dados das variáveis meteorológicas seriam utilizados para estimativas de evapotranspiração diária, é condição necessária que os dados estejam agregados em médias diárias e de forma completa, sem falhas para nenhuma das variáveis de interesse. Após a tabulação dos dados diários, retiraram-se as falhas para o cálculo da representatividade da série (ver Tabela 1) para os dois períodos avaliados. As referidas representatividades calculadas em porcentagem são para os dados contíguos diários para as variáveis: temperatura (média compensada, máxima e mínima), intensidade do vento, insolação e umidade relativa. 
Tabela 1 - Coordenadas geográficas, altitude e a representatividade da série temporal para as 27 estações meteorológicas do Estado de Minas Gerais.

\begin{tabular}{|c|c|c|c|c|c|c|c|}
\hline \# & MSR & ESTAÇÃO & LAT & $\operatorname{LON}\left({ }^{\circ}\right)$ & ALT (m) & $\begin{array}{c}\text { Dados } \\
(\%) \\
81-10\end{array}$ & $\begin{array}{c}\text { Dados } \\
(\%) \\
11-17\end{array}$ \\
\hline 1 & \multirow{2}{*}{$\begin{array}{l}\text { Campo das } \\
\text { Vertentes }\end{array}$} & Barbacena & $-21,25$ & $-43,76$ & 1126 & 77 & 27 \\
\hline 2 & & Lavras & $-21,22$ & -45 & 918 & 77 & 99 \\
\hline 3 & \multirow{3}{*}{ Zona da Mata } & Caparaó & $-20,51$ & $-41,9$ & 843 & 57 & 99 \\
\hline 4 & & Juiz de Fora & $-21,76$ & $-43,35$ & 940 & 78 & 77 \\
\hline 5 & & Viçosa & $-20,75$ & $-42,85$ & 690 & 71 & 65 \\
\hline 6 & \multirow{2}{*}{$\begin{array}{c}\text { Sul/Sudoeste } \\
\text { de Minas }\end{array}$} & Machado & $-21,66$ & $-45,91$ & 873 & 76 & 97 \\
\hline 7 & & São Lourenço & $-22,1$ & $-45,01$ & 953 & 76 & 99 \\
\hline 8 & \multirow{4}{*}{$\begin{array}{c}\text { Triângulo } \\
\text { Mineiro/Alto } \\
\text { Parnaíba }\end{array}$} & Araxá & $-19,6$ & $-46,93$ & 1023 & 83 & 97 \\
\hline 9 & & Capinópolis & $-18,71$ & $-49,55$ & 620 & 79 & 86 \\
\hline 10 & & Uberaba & $-19,73$ & $-47,95$ & 737 & 76 & 97 \\
\hline 11 & & Patos de Minas & $-18,51$ & $-46,43$ & 940 & 73 & 92 \\
\hline 12 & $\begin{array}{l}\text { Oeste de } \\
\text { Minas }\end{array}$ & Bambuí & $-20,03$ & -45 & 661 & 77 & 87 \\
\hline 13 & \multirow{3}{*}{$\begin{array}{c}\text { Metropolitana } \\
\text { de Belo } \\
\text { Horizonte }\end{array}$} & Belo Horizonte & $-19,93$ & $-43,91$ & 915 & 86 & 99 \\
\hline 14 & & $\begin{array}{c}\text { Conceição Mato } \\
\text { Dentro }\end{array}$ & $-19,02$ & $-43,43$ & 652 & 72 & 56 \\
\hline 15 & & Sete Lagoas & $-19,46$ & $-44,25$ & 732 & 71 & 56 \\
\hline 16 & $\begin{array}{c}\text { Vale do Rio } \\
\text { Doce }\end{array}$ & Caratinga & $-19,73$ & $-42,13$ & 609 & 74 & 100 \\
\hline 17 & \multirow{2}{*}{$\begin{array}{c}\text { Noroeste de } \\
\text { Minas }\end{array}$} & Formoso & $-14,93$ & $-46,25$ & 840 & 63 & 65 \\
\hline 18 & & Paracatu & $-17,24$ & $-46,88$ & 712 & 60 & 98 \\
\hline 19 & \multirow{2}{*}{ Jequitinhonha } & Diamantina & $-18,23$ & $-43,64$ & 1296 & 66 & 55 \\
\hline 20 & & Pedra Azul & -16 & $-41,28$ & 648 & 78 & 49 \\
\hline 21 & \multirow{7}{*}{$\begin{array}{l}\text { Norte de } \\
\text { Minas }\end{array}$} & Janaúba & $-15,78$ & $-43,3$ & 516 & 64 & 65 \\
\hline 22 & & Januária & $-15,45$ & -44 & 473 & 63 & 99 \\
\hline 23 & & Juramento & $-16,77$ & $-43,66$ & 648 & 55 & 76 \\
\hline 24 & & Monte Azul & $-15,08$ & $-42,75$ & 613 & 69 & 66 \\
\hline 25 & & Montes Claros & $-16,68$ & $-43,84$ & 652 & 62 & 66 \\
\hline 26 & & Pirapora & $-17,35$ & $-44,91$ & 505 & 82 & 69 \\
\hline 27 & & Salinas & $-16,15$ & $-42,28$ & 471 & 82 & 100 \\
\hline
\end{tabular}

A Figura 1, abaixo, apresenta a distribuição espacial das estações meteorológicas do INMET utilizadas nessa análise enumeradas de acordo com a Tabela 1. 


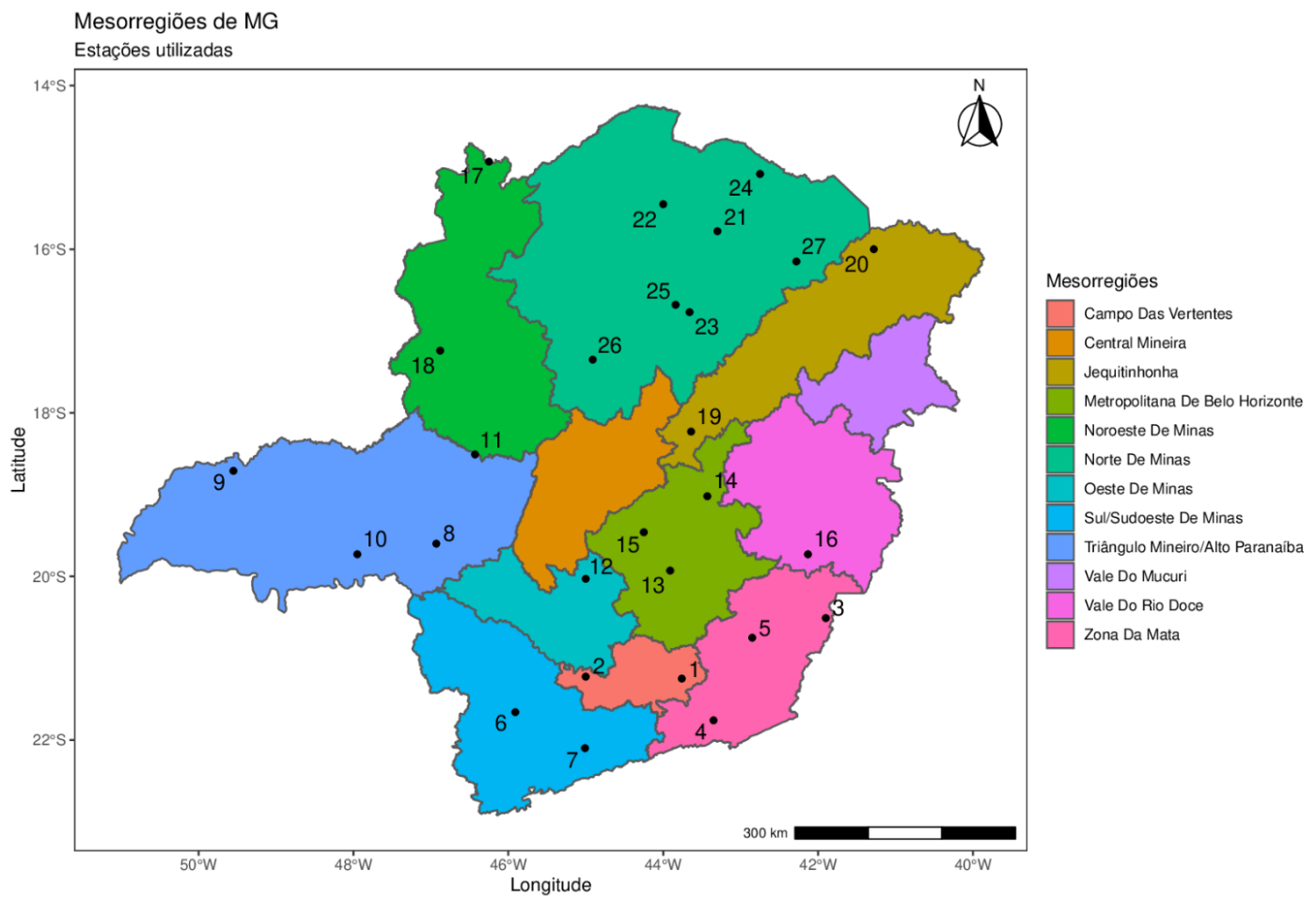

Figura 1 - Distribuição espacial das estações meteorológicas do INMET localizadas no Estado de Minas Gerais.

\subsection{ANÁLISE DE DADOS}

Uma análise de tendência foi realizada objetivando a identificação de aumentos ou decréscimos significativos nos valores de temperatura mínima, temperatura máxima, insolação, umidade relativa, temperatura média compensada e velocidade do vento. Para a realização desta análise, o método escolhido foi o Mann-Kendall (MK), uma vez que, por meio deste, as tendências podem ser classificadas como estatisticamente significativas ou não significativas (DINPASHOH et al., 2011; SALVIANO, GROPPO E PELLEGRINO, 2016). De acordo com estudo realizado (FOLHES E FISCH, 2006), aumentos e decrescimentos não sazonais (monotônicas) podem ser estimados e verificados de forma robusta por este teste.

Neste trabalho consideraram-se tendências significativas com valor da estatística "p" inferior a 5\% (FERREIRA et al., 2017; LOPO et al., 2015). Dessa forma, valores da estatística " $p$ " superiores a este limite foram desconsiderados por não mostrar significância estatística.

O tratamento de dados foi realizado por programação em ambiente $R$, tendo sido utilizados os seguintes pacotes: openair (CARSLAW E ROPKINS, 2012), lubridate (GROLEMUND E WICKHAM, 2011) e trend (POHLERT, 2020). O pacote openair disponibiliza ferramentas que permitem o manuseio mais simplificado de dados meteorológicos como aqueles oriundos das estações meteorológicas convencionais; o pacote lubridate traz funções que facilitam o trabalho com dados que envolvam datas; o pacote trend permite o estudo de 
tendências estatísticas baseadas em conjuntos de dados, e a determinação de valores estatisticamente significativos.

A expressão matemática para os cálculos dos parâmetros da tendência são descritos abaixo:

$$
S=\sum_{k=1}^{n-1} \sum_{j=k+1}^{n} \operatorname{snl}\left(x_{j}-x_{k}\right)
$$

Em que $S$ diz respeito à variável estatística; $n$, o número de elementos que compõem a série; $j$ e $k$, são os indicadores de posição da série; $s n l$, referese ao sinal da diferença dos valores da série $\left(x_{j}\right.$ e $\left.x_{k}\right)$. Deste modo, para o snl, que indica o sinal da diferença $x_{j}-x_{k}$, os seguintes limites são observados (MOREIRA E NAGHETTINI, 2016; WILKS, 2005):

$$
\operatorname{snl}\left(x_{j}-x_{k}\right)=\left\{\begin{array}{c}
+1, \text { se }\left(x_{j}-x_{k}\right)>0 \\
0, \text { se }\left(x_{j}-x_{k}\right)=0 \\
-1, \text { se }\left(x_{j}-x_{k}\right)<0
\end{array}\right.
$$

Em seguida, calcula-se a variância, que é dada pela seguinte fórmula:

$$
\operatorname{VAR}(S)=\frac{n(n-1) \cdot(2 n+5)}{18}
$$

Ao final, através da distribuição normal $Z$, prossegue-se a análise com a determinação do valor de Z:

$$
Z=\left\{\begin{array}{l}
\frac{S-1}{\sqrt{V A R(S)}} \text { se } S>0 \\
0 \text { se } S=0 \\
\frac{S-1}{\sqrt{V A R(S)}} \text { se } S<0
\end{array}\right.
$$

Para a análise realizada no artigo, o valor de $\mathrm{p}$ definido como limiar de aceitação foi de $5 \%$ e o intervalo de confiança de $95 \%$, com Z variando de -1.96 a +1.96. Logo, para toda análise cujo valor de $p$ foi inferior a 0.05 , pode-se considerar a presença de tendência monotônica.

\subsection{ESTIMATIVA DE EVAPOTRANSPIRAÇÃO DE REFERÊNCIA (ETO)}

Após o tratamento dos dados brutos, utilizou-se o software REF-ET (ALLEN, 2013; FERNANDES et al., 2012) para fornecer cálculos padronizados de 
evapotranspiração de referência baseados nas medições de dados meteorológicos. O método utilizado foi o de Penman-Monteith, cuja equação é

$$
\text { ETo }=\frac{0,408 \Delta\left(R_{n}-G\right)+Y \frac{900}{r_{\text {med }}+273,16} U_{2} *\left(e_{s}\right)-\left(e_{a}\right)}{\Delta+Y\left(1+0,34 U_{2}\right)}
$$

Em que: "Rn" é o saldo de radiação $\left(M J \cdot \mathrm{m}^{-2} \cdot \mathrm{d}^{-1}\right)$, "G" é a densidade do fluxo de calor no solo, " $U_{2}$ " é a velocidade do vento, média diária $\left(\mathrm{m} . \mathrm{s}^{-1}\right)$, " $\mathrm{T}_{\text {méd" }}$ é a temperatura média do ar $\left({ }^{\circ} \mathrm{C}\right)$, "es" é a pressão de saturação do vapor (kPa), "ea" é a pressão atual do vapor (kPa) e "y" é o fator psicrométrico $\left(\mathrm{MJ} \cdot \mathrm{kg}^{-1}\right)$.

\subsection{DETERMINAÇÃO DAS ANOMALIAS DE EVAPOTRANSPIRAÇÃO E DA EVAPOTRANSPIRAÇÃO DE VERÃO (ETV)}

As anomalias referidas nesse estudo consistem na diferença absoluta das médias mensais dos períodos estudados, levando-se em consideração a normal climatológica de 30 anos (1981-2010) e o período mais recente, de 2011 a 2017. Optou-se por utilizar o método de Penman-Monteith (PM), por ser este o recomendado pela FAO (ALLEN et al., 1998). A determinação da ETV se deu pelo somatório da evapotranspiração dos meses do verão (trimestre DJF - dezembro, janeiro e fevereiro), que é de especial importância uma vez que esta é a estação que têm a maior parcela da evapotranspiração anual. Cabe ressaltar que o estudo específico da ETv se dá pela sua importância e utilização como critério de classificação climática, como por exemplo a de Thronthwaite e Matter (CAMARGO E CAMARGO, 2000; GIMÉNEZ E GARCÍA PETILLO, 2011).

\section{RESULTADOS E DISCUSSÃO}

\subsection{ANÁLISE DE TENDÊNCIAS DAS VARIÁVEIS METEOROLÓGICAS}

Na Tabela 2 estão apresentados os resultados do teste Mann-Kendall para o período de 2011-2017 para temperatura máxima ( $T_{\text {máx }}$ ), temperatura mínima $\left(T_{\min }\right)$, temperatura média compensada ( $\left.T_{\text {média }}\right)$, umidade relativa (UR), insolação $(n)$ e velocidade do vento $(v)$. 
Tabela 2 - Tendências temporais monotônicas (não sazonais) das estações meteorológicas do INMET no Estado de Minas Gerais para o período de 2011 a 2017.

\begin{tabular}{|c|c|c|c|c|c|c|c|}
\hline Estações & $\begin{array}{c}\mathbf{n}_{\text {obs }} \\
\#\end{array}$ & $\begin{array}{c}\mathbf{T}_{\text {máx }} \\
{ }^{\circ} \mathrm{C} . \text { ano }^{-1}\end{array}$ & $\begin{array}{c}\mathbf{T}_{\text {mín }} \\
{ }^{\circ} \mathrm{C} \cdot \text { ano }^{-1}\end{array}$ & $\begin{array}{c}\mathbf{T}_{\text {média }} \\
{ }^{\circ} \mathrm{C} . \text { ano }^{-1}\end{array}$ & $\begin{array}{c}\text { UR } \\
\% \text { ano }^{-1}\end{array}$ & $\begin{array}{c}\mathbf{n} \\
\min . \mathrm{ano}^{-1}\end{array}$ & $\begin{array}{c}\mathbf{V} \\
\mathrm{m} \cdot \mathrm{s}^{-} \\
{ }^{1} \cdot \mathrm{ano}^{-1}\end{array}$ \\
\hline Barbacena $^{1}$ & 679 & $-0,95$ & $-1,51$ & $-1,04$ & NS & NS & 0,11 \\
\hline Lavras $^{1}$ & 2533 & 0,1 & NS & 0,09 & NS & NS & $-0,12$ \\
\hline Caparaó $^{2}$ & 2538 & NS & NS & 0,07 & $-0,26$ & NS & $-0,04$ \\
\hline Juiz de Fora ${ }^{2}$ & 1957 & 0,2 & 0,09 & 0,14 & $-1,1$ & NS & NS \\
\hline Viçosa² & 1673 & 0,16 & NS & 0,12 & $-0,77$ & 11,15 & $-0,07$ \\
\hline Machado $^{3}$ & 2471 & 0,15 & NS & 0,09 & NS & NS & 0,11 \\
\hline São Lourenço ${ }^{3}$ & 2526 & 0,08 & NS & 0,07 & $-0,29$ & NS & NS \\
\hline Araxá $^{4}$ & 2492 & $-0,04$ & 0,05 & NS & $-0,68$ & NS & NS \\
\hline Capinópolis ${ }^{4}$ & 2192 & 0,19 & 0,07 & 0,14 & $-0,75$ & NS & NS \\
\hline Uberaba $^{4}$ & 2484 & 0,1 & 0,07 & 0,13 & $-0,76$ & NS & 0,09 \\
\hline Patos de Minas ${ }^{4}$ & 2360 & 0,18 & 0,12 & 0,14 & $-0,84$ & NS & 0,03 \\
\hline Bambuí $^{5}$ & 2237 & 0,2 & 0,1 & 0,11 & NS & 7,47 & NS \\
\hline Belo Horizonte 6 & 2541 & 0,12 & 0,08 & 0,11 & $-0,58$ & NS & $-0,02$ \\
\hline C. Mato Dentro ${ }^{6}$ & 1441 & 0,26 & NS & 0,28 & $-0,86$ & NS & NS \\
\hline Sete Lagoas ${ }^{6}$ & 1419 & 0,46 & $-0,17$ & 0,26 & $-1,53$ & 7,76 & 0,07 \\
\hline Caratinga $^{7}$ & 2549 & 0,11 & 0,08 & 0,11 & $-0,66$ & NS & $-0,03$ \\
\hline Formoso $^{8}$ & 1671 & 0,12 & 0,23 & 0,21 & $-1,19$ & NS & NS \\
\hline Paracatu $^{8}$ & 2518 & 0,09 & 0,09 & 0,16 & $-0,89$ & NS & 0,08 \\
\hline Diamantina $^{9}$ & 1405 & 0,46 & 0,32 & 0,42 & $-1,92$ & 24,94 & $-0,35$ \\
\hline Pedra Azul ${ }^{9}$ & 1242 & 0,31 & 0,29 & 0,33 & $-0,7$ & NS & 0,09 \\
\hline Janaúba $^{10}$ & 1670 & 0,21 & NS & 0,18 & $-1,75$ & NS & NS \\
\hline Januária ${ }^{10}$ & 2522 & 0,29 & 0,24 & 0,29 & $-2,07$ & NS & 0,03 \\
\hline Juramento $^{10}$ & 1956 & 0,18 & 0,14 & 0,23 & $-1,56$ & $-5,12$ & NS \\
\hline Monte Azul ${ }^{10}$ & 1696 & 0,28 & 0,32 & 0,34 & $-0,96$ & 4,13 & $-0,06$ \\
\hline Montes Claros ${ }^{10}$ & 1675 & 0,24 & 0,31 & 0,33 & $-1,53$ & NS & NS \\
\hline Pirapora $^{10}$ & 1764 & 0,49 & 0,26 & 0,39 & $-2,08$ & 3,71 & 0,04 \\
\hline Salinas $^{10}$ & 2546 & 0,14 & 0,18 & 0,21 & $-0,97$ & 3,43 & 0,02 \\
\hline
\end{tabular}

Em que: 1- Campo das vertentes; 2- Zona da Mata; 3 - Sul/Sudoeste de Minas; 4 Triângulo Mineiro/Alto Parnaíba; 5 - Oeste de Minas; 6 - Metropolitana de BH; 7 - Vale do Rio Doce; 8 - Noroeste de Minas; 9 - Jequitinhonha; 10 - Norte de Minas. Todos os valores apresentados na tabela possuem valor de $p$ inferior 0,05 . Valores em negrito possuem valores de $p$ menores que 0,01 . Valores NS tiveram taxas estatisticamente não significativas $(>5 \%)$.

Foram identificadas tendências significativas de aumento anual de temperatura para mais de $90 \%$ das estações meteorológicas. Em relação à temperatura máxima, a estação de Pirapora é a que apresenta a maior tendência de aumento anual $\left(0,49^{\circ} \mathrm{C}^{\circ} \mathrm{ano}^{-1}\right)$, seguida das estações de Sete Lagoas e Diamantina, ambas com $0,46^{\circ} \mathrm{C}$.ano ${ }^{-1}$. Tratando-se das tendências de queda, apenas 2 estações (Araxá e Barbacena) apresentaram decréscimo $(-0,04$ ${ }^{\circ} \mathrm{C}$.ano ${ }^{-1}$ e $-0,95{ }^{\circ} \mathrm{C} . \mathrm{ano}^{-1}$, respectivamente). Tanto para a temperatura mínima quanto para a temperatura média compensada, a tendência de aumento 
também prevalece; apenas as estações de Sete Lagoas e Barbacena apresentam tendência de queda em suas temperaturas mínimas e médias compensadas. Cabe ressaltar que, apesar dos valores de tendência negativos serem estatisticamente significativos a $1 \%$ de significância no município de Barbacena, há menos de 30\% da série total de 2011 a 2017, devido a falhas na série histórica.

Levando-se em consideração a variável umidade relativa (UR), ao contrário da tendência de aumento observada para as temperaturas média compensada, máxima e mínima, há uma tendência de queda para todas as estações meteorológicas levantadas no estudo. A maior tendência de decréscimo pode ser observada na estação de Pirapora $\left(-2,08 \%\right.$.ano $\left.{ }^{-1}\right)$, seguido das estações de Januária $\left(-2,07 \% . a_{0}{ }^{-1}\right)$ e Diamantina $\left(-1,92 \%\right.$. ano $\left.^{-1}\right)$. Pressupondo pressão de vapor de água constante, a temperatura se comporta como variável inversa da umidade relativa, o que torna o resultado razoável para o mesmo período. Com o aumento das temperaturas em todo o estado, observa-se diminuição da umidade relativa do ar; tal relação é influenciada pelos calores específicos da água e do ar e pelo aquecimento homogêneo de ambos (ALVES E BIUDES, 2012; THOMAZINI, 2015). Tratando-se da variável temperatura, outros autores constataram uma realidade semelhante com tendências positivas e negativas para umidade relativa (MARENGO, 2007; SALVIANO, GROPPO E PELLEGRINO, 2016; SANCHES et al.,2017).

Tratando-se da insolação, que diz respeito às horas de brilho solar, o teste Mann-Kendall mostrou tendência estatisticamente significativa para apenas oito das 27 localidades avaliadas. A estação que apresentou a maior tendência positiva foi a de Diamantina $\left(24,97\right.$ min.ano $\left.^{-1}\right)$ seguida de Viçosa $\left(11,15 \mathrm{~min} \mathrm{ano}^{-1}\right)$. Nesta análise, apenas uma estação (Juramento) apresentou tendência negativa $\left(-5,12 \mathrm{~min} \mathrm{ano}^{-1}\right)$. Tais tendências positivas mostram a redução da cobertura de nuvens, o que está relacionado às recorrentes secas presenciadas pelo estado ao longo dos últimos anos, conforme constatado por outros trabalhos (NATIVIDADE, GARCIA E TORRES, 2017).

A velocidade do vento não apresentou um padrão nítido de tendência positiva ou negativa no estado. Dentre as estações que apresentaram as maiores tendências de aumento, encontram-se Barbacena e Machado (ambas com $\left.0,11 \mathrm{~m} . \mathrm{s}^{-1}\right)$ seguidas de Pedra Azul $\left(0,09 \mathrm{~m} \cdot \mathrm{s}^{-1}\right)$. Dentre aquelas com tendências de diminuição, estão: Lavras $\left(-0,12 \mathrm{~m} \cdot \mathrm{s}^{-1}\right)$ e Monte Azul $\left(-0,06 \mathrm{~m} . \mathrm{s}^{-}\right.$ ${ }^{1}$ ). Pode-se dizer que o vento é um importante fator atenuante da sensação térmica, estabelecendo-se nesse contexto, uma relação inversamente proporcional para com a temperatura.

\subsection{ANOMALIAS DE EVAPOTRANSPIRAÇÃO DE REFERÊNCIA}

$\mathrm{Na}$ figura 2 encontram-se os dados de anomalia mensal de evapotranspiração de referência para o período mais recente (2011 - 2017) em relação ao período climatológico (1981 - 2010), em mm.mês ${ }^{-1}$. 


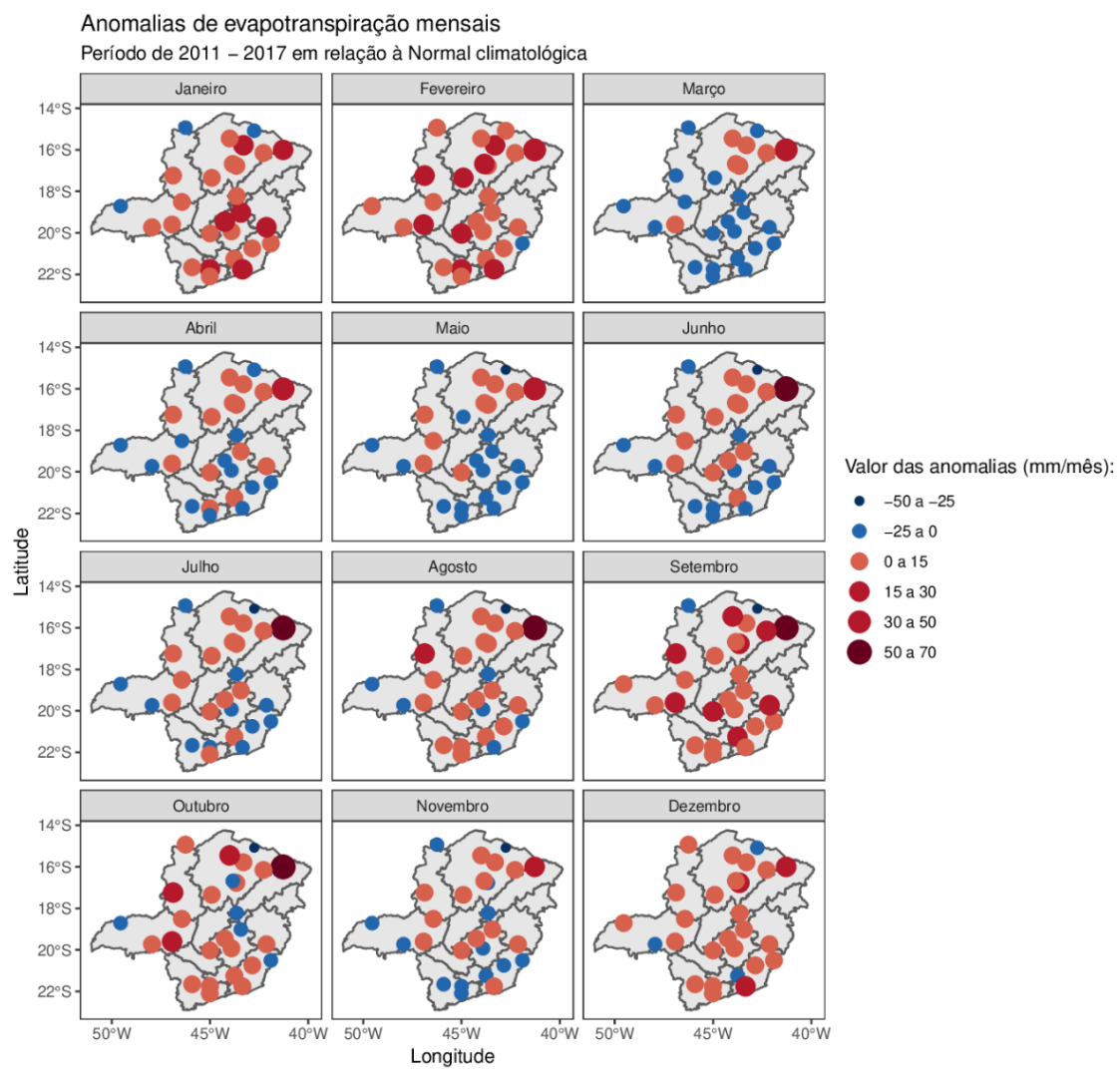

Figura 2 - Anomalias mensais de evapotranspiração de referência ( $\mathrm{mm} \cdot \mathrm{mês}^{-1}$ ) pelo método de Penman-Monteith para o período de 2011-2017 em relação à normal de 1981 $-2010$.

Na figura 2, observa-se que a maior parte das estações apresentou anomalia positiva nos meses de verão (DJF) e fim de inverno/início da primavera (ASO). Outra importante constatação refere-se às anomalias positivas em algumas estações da região norte do estado prevalecendo praticamente em todos os meses do ano, como Januária, Juramento, Janaúba, Montes Claros, Pirapora e Salinas. Tais resultados reforçam os estudos de modelagem climática na mesorregião Norte de Minas a possibilidade de climas mais quentes e secos até o fim do século XXI (REBOITA et al., 2017). Além do último, cabe destacar o trabalho de Martins e Santos (2018) que desenvolveram um estudo sobre cenários de mudanças climáticas para o estado de Minas Gerais até 2100 . De acordo com os autores as projeções são de possível aumento de até $5^{\circ} \mathrm{C}$ na temperatura do ar para algumas localidades. Mesmo no cenário mais brando (RCP 2.6), os autores verificaram possibilidade de mudanças para tipos climáticos mais quentes e áridos, principalmente nas regiões Norte, Nordeste, Jequitinhonha Noroeste e Central Mineira.

As maiores anomalias positivas foram de $10,13 \mathrm{~mm} . \mathrm{mês}^{-1}$ para São Lourenço, no Sul/Sudoeste de Minas, para setembro, e de 20,24 mm.mês ${ }^{-1}$ para Juiz de Fora, na Zona da Mata, em janeiro. As maiores anomalias negativas (em módulo) foram obtidas para Monte Azul no Norte de Minas de $-45,22$ mm.mês ${ }^{-1}$ em agosto, e 65,80 mm.mês ${ }^{-1}$ para Pedra Azul, em setembro, no Jequitinhonha. Destaca-se também maior variabilidade das anomalias da evapotranspiração para as mesorregiões Norte de Minas e Jequitinhonha, obtendo variações de até 
$43 \mathrm{~mm} \cdot \mathrm{mês}^{-1}$ entre meses na estação de Pedra Azul e variações de até 20 $\mathrm{mm}$. mês ${ }^{-1}$ na estação de Pirapora.

Em contrapartida, observam-se anomalias negativas nos meses e março até junho nas mesorregiões Sul/Sudoeste de Minas e Zona da Mata, o que evidencia menor demanda atmosférica por vapor d'água no período de 20112017, em comparação com as normais climatológicas da região. Cabe ressaltar que essas mesmas mesorregiões são mais atingidas por sistemas frontogenéticos e circulações de grande escala como as ZCOU (Zonas de Convergência de Umidade) e ZCAS (Zonas de Convergência do Atlântico Sul) que contribuem para um regime hídrico diferenciado dentro de Minas Gerais (Góis et al., 2013). Tais sistemas transientes (ciclones) são responsáveis por transporte de vapor d'água até a região Sul de Minas Gerais.

A análise de anomalias e tendência para a evapotranspiração de verão (ETV) encontram-se na Tabela 3. 
Tabela 3 - Valores de evapotranspiração de verão (ETV), anomalias e tendências temporais monotônicas para o período de 2011 a 2017.

\begin{tabular}{|c|c|c|c|c|c|}
\hline Estações & $\begin{array}{c}\mathbf{n}_{\text {obs }} \\
\#\end{array}$ & $\begin{array}{l}\mathbf{E T}_{\mathbf{v}} \\
\mathrm{mm}\end{array}$ & $\begin{array}{l}\text { Anomalias } \\
\text { mm.mês }\end{array}$ & $\begin{array}{l}\mathbf{z} \\
*\end{array}$ & $\begin{array}{c}\text { Taxas } \\
\text { monotônicas } \\
*\end{array}$ \\
\hline Barbacena $^{1}$ & 679 & 339,4 & 2,3 & $-1,0$ & $-0,084$ \\
\hline Lavras $^{1}$ & 2533 & 428,3 & 15,1 & $-1,4$ & $-0,018$ \\
\hline Caparaó $^{2}$ & 2538 & 384,7 & 5,1 & 0,7 & 0,008 \\
\hline Juiz de Fora ${ }^{2}$ & 1957 & 406,6 & 18,2 & 1,8 & 0,037 \\
\hline Viçosa ${ }^{2}$ & 1673 & 393,8 & 10,0 & 1,0 & 0,022 \\
\hline Machado $^{3}$ & 2471 & 356,8 & 8,9 & 4,9 & 0,056 \\
\hline São Lourenço ${ }^{3}$ & 2526 & 388,7 & 9,0 & 0,9 & 0,010 \\
\hline Araxá 4 & 2492 & 406,5 & 15,1 & 0,9 & 0,010 \\
\hline Capinópolis ${ }^{4}$ & 2192 & 397,0 & 0,7 & 1,8 & 0,026 \\
\hline Uberaba $^{4}$ & 2484 & 393,5 & 0,6 & 4,8 & 0,069 \\
\hline Patos de Minas ${ }^{4}$ & 2360 & 380,4 & 7,4 & 4,4 & 0,054 \\
\hline Bambuí $^{5}$ & 2237 & 353,9 & 12,6 & 4,8 & 0,056 \\
\hline Belo Horizonte 6 & 2541 & 405,6 & 6,0 & 1,6 & 0,017 \\
\hline $\begin{array}{l}\text { C. Mato } \\
\text { Dentro }\end{array}$ & 1441 & 411,0 & 12,3 & 3,5 & 0,106 \\
\hline Sete Lagoas ${ }^{6}$ & 1419 & 416,8 & 11,0 & 4,6 & 0,135 \\
\hline Caratinga $^{7}$ & 2549 & 428,9 & 13,3 & 1,4 & 0,018 \\
\hline Formoso $^{8}$ & 1671 & 398,6 & 5,3 & 3,0 & 0,065 \\
\hline Paracatu $^{8}$ & 2518 & 432,9 & 12,1 & 4,8 & 0,065 \\
\hline Diamantina $^{9}$ & 1405 & 359,7 & 3,9 & $-1,3$ & $-0,032$ \\
\hline Pedra Azul ${ }^{9}$ & 1242 & 506,1 & 31,8 & 2,9 & 0,101 \\
\hline Janaúba $^{10}$ & 1670 & 460,6 & 13,8 & 3,4 & 0,085 \\
\hline Januária ${ }^{10}$ & 2522 & 460,1 & 10,4 & 7,1 & 0,102 \\
\hline Juramento $^{10}$ & 1956 & 414,9 & 10,9 & 2,7 & 0,044 \\
\hline Monte Azul ${ }^{10}$ & 1696 & 420,3 & $-4,0$ & 0,7 & 0,021 \\
\hline Montes Claros $^{10}$ & 1675 & 452,4 & 14,2 & 2,8 & 0,065 \\
\hline Pirapora $^{10}$ & 1764 & 449,1 & 12,0 & 4,8 & 0,109 \\
\hline Salinas ${ }^{10}$ & 2546 & 436,6 & 11,9 & 3,3 & 0,044 \\
\hline
\end{tabular}

Em que: 1- Campo das vertentes; 2- Zona da Mata; 3 - Sul/Sudoeste de Minas; 4 Triângulo Mineiro/Alto Parnaíba; 5 - Oeste de Minas; 6 - Metropolitana de BH; 7 - Vale do Rio Doce; 8 - Noroeste de Minas; 9 - Jequitinhonha; 10 - Norte de Minas. Todos os valores apresentados na tabela possuem valor de $p$ inferior 0,05 . Valores em negrito possuem valores de $p$ menores que 0,01 .

De acordo com a Tabela 3, os dados de 15 das 27 estações analisadas apresentaram resultados estatisticamente significativos ( $p<0,05)$. Dentre esses resultados, observa-se congruência entre as anomalias e suas tendências. Em outras palavras, os sinais tanto da anomalia da ETv, e suas taxas monotônicas mostram-se positivos para $55 \%$ das estações. Ressalta-se que a convergência entre as tendências apresentadas indicam maior demanda 
atmosférica por vapor d'água para as estações destacadas no estado de Minas Gerais de 2011 a 2017.

Em relação às mesorregiões do estado, pode-se notar que a o Norte de Minas (10) é a que apresenta maior número de tendências positivas ( $83 \%$ das estações). Os maiores valores de anomalias encontram-se nas estações de Montes Claros $\left(14,2 \mathrm{~mm} . \mathrm{mês}^{-1}\right)$ e Janaúba $\left(13,8 \mathrm{~mm} \cdot \mathrm{mês}^{-1}\right)$, enquanto que o menor valor observado se refere à estação de Januária $\left(10,4 \mathrm{~mm} \cdot \mathrm{mês}^{-1}\right)$. Cabe ressaltar o estudo de REBOITA et al.(2017), em que projetam-se climas mais quentes e secos no Norte de Minas para o período de 2011 a 2100. Dessa forma, os sinais de anomalias presentes nas mesorregiões supracitadas corroboram com a projeção, e mostram que o sinal positivo já se mostra a partir do período de 2011 até 2017.

Em relação ao Noroeste de Minas (8), todas as estações avaliadas apresentaram valores considerados estatisticamente significativos. O maior valor observado de anomalia pertence à Paracatu $\left(12,1 \mathrm{~mm}\right.$. mês $\left.{ }^{-1}\right)$ e o menor a Formoso $\left(5,3 \mathrm{~mm}\right.$. mês $\left.^{-1}\right)$. Tais valores também apresentam correlação, pois, uma vez que a região do Noroeste de Minas faz fronteira com o Norte Minas, é razoável expressarem características semelhantes.

Cabe destacar o Triângulo Mineiro e Alto Parnaíba (4), os quais apresentaram valores de $\mathrm{p}$ menores que 0,05 (Patos de Minas com 7,4 mm.mês ${ }^{1}$ e Uberaba com 0,6 mm. mês ${ }^{-1}$ ); região metropolitana de Belo Horizonte (6), na qual, as estações, Conceição do Mato Dentro e Sete Lagoas apresentaram valores de $12,3 \mathrm{~mm} \cdot \mathrm{mês}^{-1}$ e $11,0 \mathrm{~mm} \cdot \mathrm{mês}^{-1}$, respectivamente. As demais estações apresentaram apenas uma tendência estatisticamente significativa: Jequitinhonha (9) (Pedra Azul com 31,8 mm. mês ${ }^{-1}$ ), Sul/Sudoeste de Minas (3) (Machado com 8,9 mm.mês ${ }^{-1}$ ) e, por fim, a mesorregião do Oeste de Minas (5), com apenas uma estação (Bambuí com 12,6 mm.mês ${ }^{-1}$ ).

De forma geral, as mesorregiões localizadas ao norte do estado Minas Gerais são as mais representativas, no sentido de apresentarem resultados coincidentes entre as estações meteorológicas ali inseridas, com tendências estatisticamente significativas, além de apresentarem os maiores valores de evapotranspiração de verão observados (Pedra Azul - 506,1 mm, Janaúba 460,6 mm e Januária - 460,1 mm).

\section{CONCLUSÃO}

Os resultados das análises de tendência para variáveis meteorológicas de 27 estações meteorológicas convencionais de Minas Gerais para o período de 2011 a 2017, evidenciaram comportamentos importantes. Dentre as tendências monotônicas das variáveis meteorológicas, cabe destacar a temperatura máxima, para a qual as maiores tendências de aumento pertencem às estações

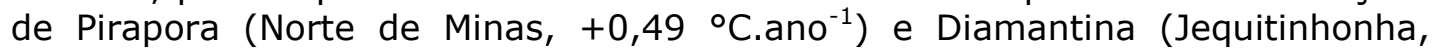
$+0,46^{\circ} \mathrm{C}$. ano ${ }^{-1}$ ). Padrão semelhante ao da temperatura máxima, foi observado para as temperaturas médias compensada e mínimas. A umidade relativa apresentou tendência negativa $\left(\% \cdot\right.$ ano $^{-1}$ ) em $96 \%$ das estações avaliados; 0 maior decréscimo pertenceu a estação de Pirapora $\left(-2,08 \%\right.$. ano $\left.{ }^{-1}\right)$ seguido de Januária $\left(-2,07 \%\right.$. ano $\left.{ }^{-1}\right)$. 
A análise de anomalias para a evapotranspiração de referência no verão mostrou valores positivos principalmente para as mesorregiões do Norte de Minas, Noroeste, Oeste de Minas e Jequitinhonha. Para essas, o teste MannKandall para a evapotranspiração de verão indica sinais congruentes com as anomalias calculadas e suas respectivas tendências, sendo estatisticamente significativas. Cabe destacar os valores das lâminas de evapotranspiração nos meses mais quentes do ano para as estações de Janaúba $(460,6 \mathrm{~mm})$ e Januária $(460,1 \mathrm{~mm})$, além disso, as mesmas apresentaram tendência positiva de aumento no período recente. Tais perdas, associadas a tendências significativas de aumento ao longo dos anos tem implicações diretas no setor de agricultura dessas regiões.

Estudos relativos às mudanças climáticas para o período de 2011 a 2100 indicam alterações nas características climáticas nas mesorregiões Norte de Minas, Noroeste de Minas e Jequitinhonha, com maiores perdas hídricas e climas mais secos. Em consonância, os dados meteorológicos do período de 2011 a 2017 já registram tendências significativas de aumento de temperatura e diminuição de umidade relativa, destacando-se que os maiores valores positivos de anomalias de evapotranspiração se encontram nas regiões supracitadas. Dessa forma, cabe destacar o papel importante do monitoramento de anomalias meteorológicas no estado de Minas Gerais, a fim de fomentar estratégias de mitigação de mudanças climáticas, estresse hídrico e manejo de água no solo.

\section{REFERÊNCIAS BIBLIOGRÁFICAS}

ALENCAR, L. P. DE.; SEDIYAMA, G. C.; MANTOVANI, E. C.; MARTINEZ, M. A. Tendências recentes nos elementos do clima e suas implicações na evapotranspiração da cultura do milho em Viçosa - MG. Engenharia Agrícola, v. 31, n. 4, p. 631-642, 2011.

ALENCAR, L. P. DE; SEDIYAMA, G. C.; MANTOVANI, E. C. Estimativa da evapotranspiração de referência (ETO padrão FAO), para Minas Gerais, na ausência de alguns dados climáticos. Revista Engenharia Agrícola, v. 35, n. 1, p. 1809-4430, 2015.

ALLEN, R. REF-ET: Reference Evapotranspiration Calculation Software. University of Idaho Research and Extension Center, p. 99, 2013.

ALLEN, R. G.; PEREIRA, L. S.; RAES, D.; SMITH, M. Crop evapotranspiration: Guidelines for computing crop water requirements. FAO irrigation and drainage paper: 56, p. 1-15, 1998.

ALMEIDA, J. B. D.; MOREIRA, A. A.; FERNANDES, F. H. S.; ALMEIDA, R. P. DE.; NERY, C. V. M. O sensoriamento remoto aplicado ao estudo da desertificação na região semiárida do Norte de Minas Gerais. Rev. Bras. Geom., v. 2, n. 2, p. 3139, 2014.

ALVARES, C. A.; STAPE, J. L. SENTELHAS, P. C.; GONÇALVES, J. L. de M.; SPAROVEK, P. Köppen's climate classification map for Brazil. Meteorologische Zeitschrift, v. 22, n. 6, p. 711-728, 2014.

ALVES, E. D. L.; BIUDES, M. S. ANALYSIS OF AIR TEMPERATURE AND RELATIVE HUMIDITY : STUDY OF. Revista Internacional Interdisciplinar INTERthesis, v. 9, n. 2, p. 139-156, 2012. 
ÁVILA, A. Uma síntese do quarto relatório do ipcc. Revista Multiciência, n. 8, p. 163-168, 2007.

BASTOS, S. Q. DE A.; GOMES, J. E. Dinâmica da agricultura no estado de minas gerais. Análise estrutural-diferencial para o período 1994-2008. Ruris, v. 5, n. 2, p. 45-76, 2011.

BEZERRA, B. G.; SILVA, B. S.; FERREIRA, N. J. Estimativa da evapotranspiração real diária utilizando-se imagens digitais tm - landsat 5 Universidade Federal de Campina Grande (UFCG). Centro de Previsão de Tempo e Estudos Climáticos (CPTEC). Rev. Bras. de Meteorol., v. 23, n. 3, p. 305-317, 2008.

BLANK, D. M. P. O Contexto Das Mudanças Climáticas E As Suas Vítimas. Mercator, v. 14, n. 2, p. 157-172, 2015.

CAMARGO, Â. P. DE; CAMARGO, M. B. P. DE. Uma revisão analítica da evapotranspiração potencial. Bragantia, v. 59, n. 2, p. 125-137, 2000.

CARSLAW, D.; ROPKINS, K. openair - An R package for air quality data analysis. Environmental Modelling \& Software, v. 27-28, p. 52-61, 2012.

CARVALHO, L. G. DE.; RIOS, G. F. A.; MIRANDA, W. L.; NETO, P. C. Evapotranspiração De Referência: Uma Abordagem Atual De Diferentes Métodos De Estimativa. Pesquisa Agropecuária Tropical, v. 41, n. 3, p. 456-465, 2011.

COURA, S. M. D. C. Mapeamento de vegetação do estado de minas gerais utilizando dados modis. Instituto Nacional de Pesquisas Espaciais (INPE), 2007.

DENSKI, A. P. N.; BACK, Á. J. Aderência De Distribuições De Probabilidades Aos Dados De Evapotranspiração De Referencia Decendial. Revista Brasileira de Climatologia, v. 17, p. 27-40, 2015.

DINPASHOH, Y.; JHAJHARIA, D.; FAKHERI-FARD, A.; SINGH, V. P.; KAHYA, E. Trends in reference crop evapotranspiration over Iran. Journal of Hydrology, $v$. 399, n. 3-4, p. 422-433, 2011.

FAYER, G. C.; PEREIRA, J. C.; RIBEIRO, L. G.; JÚNIOR, L. B.; FERREIRA, P. M. Crise hídrica: Evolução dos decretos estaduais sobre escassez hídrica em Minas Gerais. III Simpósio de Recursos Hídricos da Bacia do Rio Paraíba do SUL, n. 1999, p. 1-10, 2017.

FERNANDES, J. M.; OLIVEIRA, J. B. DE.; SOUZA, A. L. M. DE.; SILVA, G. S. DA.; IZIDIO, N. S. DE. C. Avaliação de modelos de estimativa da evapotranspiração de referência em Campos Sales - CE. Conexão Ciência e Sociedade. p. 58-67, 2012.

FERREIRA, P. DOS S.; PEDROSO, G. V.; DOMICIANO, G. J.; MARCOS, S. A. DO.; MEIRA, S. W. DE. Avaliação da tendência espaço-temporal da precipitação pernambuco. Revista Brasileira de Climatologia, v. 21, p. 113-134, 2017.

FOLHES, M. T.; FISCH, G. Caracterização climática e estudo de tendências nas séries temporais de temperatura do ar e precipitação em Taubaté (SP) Resumo Climate assessment and trend analysis in air temperature and precipitation time series in Taubaté (SP) Abstract. Revista ambiente e água, v. 1, n. 1, p. 61-71, 2006. 
FRANCISCO, P.; PINTO, E. Perdas agrícolas para o seguro rural, efeito da água no rendimento das culturas. Curso de comprovação e perdas agrícolas para o seguro rural, proagro e proagromais, 2012.

FREITAS, A. A. DE; BEZERRA, F.; FONTENELE, F. DAS C. B. Determinação Da Evapotranspiração Máxima E Real E Do Fator De Sensibilidade Ao Déficit Hídrico Da Melancia Em Canindé, Ce. Revista Brasileira de Engenharia Agrícola e Ambiental, v. 3, n. 3, p. 298-303, 1999.

GIMÉNEZ, L.; GARCÍA PETILLO, M. Evapotranspiración de cultivos de verano para dos regiones climáticamente contrastantes de Uruguay. Agrociencia Uruguay, v. 15, n. 2, p. 100-108, 2011.

GÓIS, G.; DELGADO, R. C.; JÚNIOR, J. F. DE. O.; LYRA, G. B.; BRASILEIRO, F. G. índices de severidade de seca aplicados na produção do milho em três mesorregiões do estado de Minas Gerais, MG (S. B. de Agrometeorologia, Ed.)XVIII Congresso Brasileiro de Agrometeorologia. Anais.Belém: VII Reunião Latino Americana de Agrometeorologia, 2013.

GOMES, S. Ajuste e avaliação de um modelo de balanço hídrico decendial e estudo dos limites de sua utilização em algumas localidades no estado do paraná. Universidade Federal do Paraná - UFPE, 2005.

GROLEMUND, G.; WICKHAM, H. Dates and times made easy with lubridate. Journal of Statistical Software, v. 40, n. 3, p. 1-25, 2011.

INSTITUTO BRASILEIRO DE GEOFRAFIA E ESTATÍSTICA. Disponível em: <https://agenciadenoticias.ibge.gov.br/agencia-sala-de-imprensa/2013agencia-de-noticias/releases/22374-ibge-divulga-as-estimativas-de-populacaodos-municipios-para-2018>. Acessado 29 de abril de 2020.

IPCC. Climate Change 2014 Mitigation of Climate Change. New York: Cambridge University Press, 2014.

JÚNIOR, A. D. S. Köppen para o zoneamento climático do estado de Minas Gerais. Universidade Federal de Lavras, 2009.

KINGSTON, D. G.; TODD, M. C.; TAYLOR, R. G.; THOMPSON, J .R.; ARNELL, N. $W$. Uncertainty in the estimation of potential evapotranspiration under climate change. Geophysical Research Letters, v. 36, n. 20, p. 3-8, 2009.

KÖPPEN, W. The thermal zones of the Earth according to the duration of hot, moderate and cold periods and to the impact of heat on the organic world. Meteorologische Zeitschrift, v. 20, n. 3, p. 351-360, 2011.

KOTTEK, M. et al. World map of the Köppen-Geiger climate classification updated. Meteorologische Zeitschrift, v. 15, n. 3, p. 259-263, 2006

LEMOS FILHO, L. C. A.; CARVALHO, L. G.; EVANGELISTA, A. W. P.; ALVES, J. J. Análise espacial da influência dos elementos meteorológicos sobre a evapotranspiração de referência em Minas Gerais. Revista Brasileira de Engenharia Agrícola e Ambiental, v. 14, n. 12, p. 1294-1303, 2010.

LIMA, J. C. DE.; ARRAES, F. D. D.; OLIVEIRA, J. B. DE.; NASCIMENTO, F. A. L. DO.; MACÊDO, K. G. Parametrização da equação de Hargreaves e Samani para estimativa da evapotranspiração de referência no Estado do Ceará, Brasil1. Revista Ciência Agronômica, v. 47, n. 3, p. 447-454, 2016. 
LOPO, A. B.; DOMONKOS, P.; SPYRIDES, M. H. C.; SIGRÓ, J.; LUCIO, P. S. Método ACMANT (Adapted Caussinus-Mestre Algorithm for homogenising Networks of Temperature series): aplicação em dados de Temperatura média do ar da cidade de Aracaju-SE (Brasil). Ciência e Natura, v. 37, n. Especial, p. 8$16,2015$.

MARENGO, J. A. Mudanças Climáticas Globais e seus Efeitos sobre a Biodiversidade Caracterização do Clima Atual e Definição das Alterações Climáticas para o Território Brasileiro ao Longo do Século XXI. Ministério do Meio Ambiente, n. 2, p. 82, 2007.

MARTINS, F. B.; SANTOS, D. Classificação climática de Köppen e de Thornthwaite para Minas Gerais: Cenário atual e projeções futuras. n. November, 2018.

MENDES, R. S. Determinação da evapotranspiração por método direto e indiretos e dos coeficientes de cultura da soja para o Distrito Federal. p. 58, 2006.

MOREIRA, J. G. DO; NAGHETTINI, M. Detecção de Tendências Monotônicas Temporais e Relação com Erros dos Tipos I e II : Estudo de Caso em Séries de Precipitações Diárias Máximas Anuais do Estado do Acre Detecting Monotonous Time Trends as Related to Type I and Type II Errors: Case Study in. Revista Brasileira de Meteorologia, v. 31, n. 4, p. 394-402, 2016.

MOSTER, C.; LIMA, W. DE P.; ZAKIA, M. J. B.; CÂMARA, C. D. Determinação do ano hidrológico visando a quantificação do balanço hídrico em microbacias experimentais. Circular Técnica IPEF, n. 197, p. 1-6, 2003.

NATIVIDADE, U. A.; GARCIA, S. R.; TORRES, R. R. Tendência dos índices de extremos climáticos observados e projetados no estado de Minas Gerais. Revista Brasileira de Meteorologia, v. 32, n. 4, p. 600-614, 2017.

PENEREIRO, J. C. et al. Distribuições de Tendências Sazonais de Temperatura Média e Precipitação nos Biomas Brasileiros. Revista Brasileira de Meteorologia, v. 33, n. 1, p. 97-113, 2018.

POHLERT, T. trend: Non-Parametric Trend Tests and Change-Point Detection. [s.l: s.n.]. Disponível em: <https://cran.r-project.org/package=trend>.

REBOITA, M. S.; MARTINS, F. B.; GONZAGA, G.; SANTOS, D. F. DOS. Classificação climática de köppen e de thornthwaite para minas gerais: cenário atual e projeções futuras. Revista Brasileira de Climatologia, v. 21, n. Edição especial Dossiê Climatologia de Minas Gerais, p. 295-312, 2017.

REMROVA, M.; CÍSLEROVÁ, M. Analysis of climate change effects on evapotranspiration in the watershed uhlírská in the Jizera mountains. Soil and Water Research, v. 5, n. 1, p. 28-38, 2010.

SALVIANO, M. F.; GROPPO, J. D.; PELLEGRINO, G. Q. Análise de Tendências em Dados de Precipitação e Temperatura no Brasil Trends Analysis of Precipitation and Temperature Data in Brazil. Revista Brasileira de Meteorologia, v. 31, n. 1, p. 64-73, 2016.

SANCHES, F. D. O.; SILVA, R. V. DA.; FERREIRA, R. C.; CAMPOS, C. A. A. Climate change in the Triângulo Mineiro Region - Brazil. Revista Brasileira de Climatologia, v. 21, p. 570-587, 2017. 
SENTELHAS, P. C.; GILLESPIE, T. J.; SANTOS, E. A. Evaluation of FAO PenmanMonteith and alternative methods for estimating reference evapotranspiration with missing data in Southern Ontario, Canada. Agricultural Water Management, v. 97, n. 5, p. 635-644, 2010.

THOMAZINI, L. F. V. Universidade de São Paulo Escola Superior de Agricultura " Luiz de Queiroz " Estudo do comportamento da temperatura e da umidade relativa do ar no interior de um secador solar misto de ventilação natural Piracicaba 2015. Universidade de São Paulo - Escola Superior de Agricultura Luiz de Queiroz, 2015.

THORNTHWAITE, C. W. An Approach toward a Rational Classification of Climate American Geographical Society Stable URL : http://www.jstor.org/stable/210739 Accessed : 11-08-2016 18: 02 UTC. Geographical Review, v. 38, n. 1, p. 55-94, 1948.

WANG, H.; XU, S.; SUN, L. Effects of climatic change on evapotranspiration in Zhalong Wetland, Northeast China. Chinese Geographical Science, v. 16, n. 3, p. 265-269, 2006.

WILKS, D. Statistical methods in the atmospheric sciences. 2. ed. Cornell: Academic Press, 2005 